\title{
KEYWORDS INDEX
}

admittance plot: 401, 403, 404, 405, 407

adsorption: 30, 43, 46, 50, 79, 147, 148, 149, 205, 209, 210, 211, 212, 213, 214, 216, $218,221,222,225,226,227,228,229,230,231,232,233,234,249,255$, $256,257,258,259,260,261,263,264,265,268,269,270,271,298,323$, $363,365,366,367,368,369,371,372,373,398,419,420,425,426,427$, $428,429,432,433,434,443,447,449,453,467,468,469,471,487$

adsorption process: 46, 205, 214, 221, 230, 234, 249, 258, 261, 264, 363, 366, 368, 372 , 447

alkoxybenzenes: 285,287

aluminium: 153, 363, 364, 365, 366, 367, 368, 369, 370, 371, 466, 469

aluminium oxide: 153,466

amorphous metallic alloy: 131

amperometric detection: 383,388

anodic oxidation: $1,2,3,4,7,8,9,10,19,21,22,23,28,29,30,31,32,33,38,55,58$, $153,215,231,259,285$

anodization: 153

AOPs: 1, 2, 3, 19, 20, 23, 25, 39

ascorbic acid: $67,121,127$

atomistic model: 273, 275, 276, 277, 278, 279, 280, 281

biodegradation: $319,321,323,333$

biosensors: 131, 132, 136, 137, 174, 350

biosorption: 43, 44, 46, 47, 48, 50, 53

boron-doped diamond electrode: 1,19

brass: 471, 472, 474, 475, 476, 478

brij-30: 205, 206, 209, 210, 211, 212, 213, 214, 215, 216, 217, 218, 249

capacitance dispersion: 263, 264, 265, 266, 268, 269, 270, 271

carbon felt cathode: 1

catalysis: $19,175,263,335,336,337,340,344,345,346,347,405,419,481$

cetyl pyridinium bromide (CPB): 249, 250, 253, 261

classical model: 273, 274, 277, 281

cobalt nitroprusside: 383

composite electrode: 67

conducting materials: 285,286

conducting polymer: $55,56,349,350,352,353,354,360$

contaminants' treatment in organic solvents: 185

coordination compounds: 435

copper alloys: 453,454 
corrosion: 64, 131, 195, 196, 197, 198, 199, 200, 202, 203, 204, 205, 207, 208, 209 , $210,211,212,213,215,216,218,221,222,224,225,226,227,228,229$, $231,232,234.237,238,239,242,244,245,247,248,249,250,251,252$, $253,254,255,257,258,261,262,319,320,321,322,323,324,325,326$, $328,329,330,331,332,333,334,363,365,367,368,369,370,371,372$, $373,391,409,410,411,412,413,414,415,416,417,419,420,421,423$, $425,426,427,428,429,430,431,432,433,439,443,444,445,446,448$, $450,452,453,454,455,456,457,458,462,463,465,466,467,468,469$, $470,471,472,473,474,475,476,477,478,479,480$

corrosion inhibitor: $205,218,221,222,234,249,250,261,319,320,321,323,324$, $325,326,328,330,331,332,333,334,363,425,432,434,439$, $443,452,454,458,469,470,472$

critical nucleus size: $273,274,275,277,278,279,280,281$

current efficiencies: 435

cyclic voltammetry: $57,89,91,92,93,94,97,98,99,104,119,121,122,139,173$, $178,179,263,266,267,287,335,337,349,350,384,391,392$, 403

cysteine: 119

degradation: $1,2,6,7,8,9,10,11,12,15,16,17,21,24,25,26,27,28,32,33,34,35$, $36,37,38,40,56,91,319,320,321,323,326,331,332,333,334,358$

diesel pipeline: 319

diethienylethylenes: 89

Differential Staircase Voltammetry: 103, 104, 116

disorder: 119, 263, 264, 265, 369, 427

drugs: $19,20,23,25,32,33,38,176,419$

DSCV: 103, 104, 105, 106, 109, 110, 112, 114, 116, 117

EAOPs: 1, 3, 19, 21, 22, 23, 28, 33, 38, 39

edge plane pyrolytic graph (EPPG) electrode: 401, 402, 403, 405, 406, 407

electrical conductivity: 55, 62, 63, 163, 164, 165, 166, 167, 168, 169, 171, 172, 350, 360

electrocatalysis: $335,344,346,347,481,485$

electrochemical noise: 195, 197, 409, 410, 417

electrochemical oxidation mechanism: 89, 90, 92, 94, 97

electrochemical oxygen reduction reaction (ORR): 335, 336, 347

electrochemical synthesis: 435

electrochemistry: 2, 43, 44, 46, 53, 100, 104, 172, 185, 266, 293, 294, 296, 297, 315, $317,351,361,401,402,403,435,480$

electrocrystallization: $273,276,277,278,279,281,282$

electrodeposition: 55, 56, 57, 63, 144, 146, 148, 171, 277, 336, 338, 339, 343, 346, 350, $391,392,393,399,481,482,483,487$

electro-Fenton: $1,2,3,4,5,6,7,8,9,10,11,12,13,14,15,16,17,19,22,23,32,33$, 38,39

electrostatic effects: $43,47,51$

electrosynthesis: 285

flavour enhancer: 173,174

flow injection: 67

flow loop method: $319,328,332$

food: $80,120,173,174,175,176,181,182,183$

FT-IR spectroscopy: 208, 225, 252, 285, 286, 287, 288, 289, 319, 321, 323, 332, 350, $351,359,419,420,443,444,449$ 
galvanic current: 197, 237, 239, 242, 243, 244, 245, 453, 455, 462, 463, 464, 465, 469 geothermal brine: 409, 412, 417

giant magnetoimpedance (GMI): 131

glass carbon (GC) electrode: $335,338,339,343,401$

glutamate: $173,174,175,176,177,178,179,180,181,182,183$

gold electrode: $173,174,178,179,266,267,269,311,336$

$\mathrm{H}_{2} \mathrm{SO}_{4}: 6,12,13,16,25,205,206,207,208,209,210,211,212,213,214,215,216$, $217,221,223,224,225,226,227,228,229,230,231,232,249,250,251,252$, $253,254,255,256,257,258,259260,261,268,279,338,392,443,444,445$, $446,447,450,451,482$

heterogeneous charge transfer: 293,312

hydrochloric acid: $195,197,200,436$

hydrogen evolution reaction: 211, 227, 256, 295, 296, 396, 397, 481, 482, 483, 485, 486,487

hysteresis: 163, 168, 169, 267

impedance spectroscopy: 79, 80, 81, 83, 84, 87, 88, 263, 264, 265, 266, 268, 271, 401, 407

infra-red spectroscopy: 205, 208, 216, 221, 225, 233, 249, 252, 261

inhibition: 205, 208, 209, 210, 211, 213, 214, 216, 218, 221, 222, 224, 225, 226, 227, $229,230,231,234,249,252,253,254,255,256,257,258,260,261,319$, $320,321,322,328,329,333,334,363,364,365,366,367,370,371,372$, $419,420,421,423,424,425,427,428,429,432,443,444,445,446,449$, $453,454,465,466,467,468,469,471,472,474,477,479$

inhibitors: 205, 206, 207, 208, 209, 210, 211, 212, 213, 214, 215, 216,218, 221, 222 , $224,225,226,227,228,229,230,231,232,233,234,249,250,251,252$, $253,254,255,256,257,258,259,260,261,262,319,320,321,322,323$, $324,325,326,327,328,329,330,331,332,333,334,363,364,365,366$, $368,369,370,371,372,419,420,421,422,423,424,425,426,427,428$, $429,430,431,432,433,434,439,443,444,445,446,447,448,449,450$, $452,453,454,458,467,468,469,470,471,472,473,474$

interacting and noninteracting redox centers: 103

ionic liquids: $163,164,171,172,335,336,338,401,402$

iron tetrakis(p-sulphonatophenyl) porphyrin: 185

IR spectra: 205, 221

isomerization kinetics: 89

Langmuir adsorption isotherm: 419

Langmuir-Schaefer films: 139, 140

layer-by-layer electrodeposition: 55, 56, 57, 63

linear polarization: $409,410,412$

manganese oxide $\left(\mathrm{MnO}_{\mathrm{x}}\right): 335$

master curve: $43,49,51,52$

membrane potential: $79,80,81,82,83,86,87$

metal/solution interface: 263,319

microbiologically influenced corrosion: 319,333

microelectrodes: 103, 104, 105, 109, 112, 116

mild steel: 205, 206, 207, 208, 209, 210, 211, 212, 213, 214, 215, 216, 217, 218, 221, $222,223,224,225,226,227,228,229,230,231,232,234,249,250,251$, $252,253,254,255,256,257,258,259,260,261,333,391,392,399,419$, $420,421,422,423,426,427,428,429,430,431,432,443,444,445,446$, $447,448,450,451,467,482,483$ 
mineralization: $1,2,3,4,7,8,10,11,12,14,16,17,19,20,22,23,25,26,27,28,29$, $30,32,33,34,38$

modified electrodes: 139, 140, 146, 147, 149, 338, 339, 383, 384, 386, 389

modified glassy carbon electrode: 383

modulated complexation by electrochemistry: 185

molecular switches: $89,90,98$

molten alkali carbonates: 237, 238, 241, 242, 245, 248

molten alkali nitrates: 237, 238, 241, 242, 244, 245, 248

molybdenum: 195, 196, 203

molybdophosphoric acid: 375,376

mono azo dyes: 363, 364, 365, 366, 367, 368, 369, 370, 371, 372

monocarboxylates:453, 454, 467, 468, 469

multicenter molecules: 103, 104, 109

multi-layered film: 55, 56, 57, 58, 61, 63

multipotential techniques: 103

multi-walled carbon nanotubes (MWCNTs): 335, 336

nano-porous: 153

NaOH: 24, 28, 155, 363, 365, 366, 367, 368, 369, 370, 371, 372, 392, 481, 482, 483, 485,486

Ni-Ce alloy: 481, 482, 483, 485, 487

nimesulide: 375,376

niobium:195, 196, 198, 199, 200, 201, 202, 203, 204

nitrite oxidation: $383,386,388$

nucleation: $144,148,149,164,273,274,275,276,277,278,279,280,281,282,351$, 352

organic salts: 285

oxygen-diffusion cathode: 1

ozonation: $19,23,24,25,27,28,38$

PEGME: 221, 222, 223, 225, 226, 227, 228, 229, 230, 231, 232, 233, 234

peroxi-coagulation: $1,3,5,10,11,17$

PET-membrane: 79

pharmaceutical analysis: 375

pharmaceuticals: $1,2,20,39,80,119,127,128,375,376,377,380,381,419$

phase transition: $163,168,171,265$

phenoxides: $435,436,439,441$

photoelectro-Fenton: $3,5,7,8,9,16,17,19,23,32,33,36,38$

pitting corrosion: $131,202,237,238,239,242,244,247,248,453,454,455,456,457$, $458,462,463,465,466,467,468,469$

platinum electrode: $91,119,120,121,122,123,125,126,128,207,224,239,252,285$, $409,455,473$

PMT: 471, 472, 473, 474, 475, 476, 477, 478

polarization: $195,197,198,199,200,205,206,207,208,209,211,214,215,216,221$, $222,223,224,225,227,230,231,232,237,239,240,241,249,250,252$, $253,255,258,259,260,265,300,322,323,330,331,332,363,364,369$, $370,371,398,409,410,411,412,414,415,419,421,429,430,443,444$, $445,446,447,455,456,457,458,463,466,469,472,474,482,485,486$

polarization curves: 195, 197, 198, 199, 200, 209, 215, 223, 225, 231, 239, 240, 241, $253,259,323,330,331,369,370,409,411,414,429,430,445$, $446,447,455,456,457,458,472,485$

poly(3,4-ethylenedioxythiophene): 55 
poly(N-methylpyrrole): $55,56,349,350$

potential dependent frequency factor: 293

potentiodynamic polarization: $198,199,419,421,430,485$

potentiometric determination: 375

PVC: $67,68,69,70,71,72,73,74,75,76,77,322,375,376,377,378,379,380$

reduction: $3,4,14,19,21,23,24,56,58,60,61,63,64,86,87,92,94,103,119,120$, $122,123,124,143,144,145,146,147,148,149,150,166,173,179,181$, $183,198,203,209,210,225,226,267,295,300,303,308,330,331,332$, $335,336,337,339,340,341,344,345,346,347,354,355,356,357,358$, 383,435

PVC membrane sensor: 375,380

room temperature ionic liquids (RTILs): 335, 336, 401, 402

rotating cage method: 319,332

roughness: $263,264,265,268$

sacrificial zinc anode: 435,438

scanning electron microscopy: 80, 205, 208, 216, 221, 225, 232, 249, 252, 260, 268, $351,352,419,422,431,433,444$

SEM: 80, 205, 206, 208, 216, 221, 222, 225, 232, 237, 249, 250, 252, 260, 268, 287, $288,323,329,338,339,340,352,394,395,419,422,431,443,444,450$

silica: 195, 208, 216, 217, 225, 233, 252, 261, 262, 444, 449

sonoelectro-Fenton: $1,3,15,16,17$

specific anion adsorption: 263, 268

spherical electrodes: 103, 104, 105, 109, 110, 112, 116

stainless steel alloy: 237, 238, 239, 240, 241, 242, 243, 244, 245, 246, 247

standard rate constant: 293, 296, 310, 315, 335, 337, 341

steel carbon: 409

surface-complexation reaction: $139,149,151$

temperature: $3,21,24,26,27,28,46,80,82,86,99,105,141,153,163,164,165,166$, $167,168,169,171,172,178,195,196,197,204,205,206,207,208,209$, $210,211,212,213,214,218,221,222,224,225,226,227,228,229,230$, $234,237,238,243,244,245,246,247,249,250,251,252,253,254,255$, $256,257,259,261,266,295,299,301,311,313,321,335,336,338,349$, $350,351,363,367,368,369,372,376,384,401,402,409,411,414,415$, $416,419,420,421,424,425,427,436,437,441,444,445,446,448,449$, $450,473,484,485$

temperature influence: 153

tetra-N-butylammonium iodide (TBAI): 443, 445, 448, 450

thiadiazoles: 419, 420, 423, 427, 429, 430, 432, 433

tosylate anion: $349,350,355,356$

triethanolamine: 391, 392, 393, 398, 399

TTF-TCNQ: $67,68,69,70,71,72,73,74,75,76,77$

voltammetry: $57,89,91,92,93,94,97,98,99,103,104,116,119,121,122,124,125$, $139,173,178,179,263,266,267,287,335,337,349,350,384,391,392$, 403

XPS: $79,80,81,86,87$

XRD: 132, 391, 394, 395, 396

Zn-Ni: 391, 392, 394, 395, 396 